\title{
Lieder im DaF-Unterricht - Konzepte und Lehrmaterialien
}

\author{
Andrea Schmitt
}

\section{Vorbemerkung}

Musik ist universell und in der einen oder anderen Form in allen Kulturen zuhause, daher gehören Musik und Lieder zum Leben fast aller Menschen. Als ein Teil der Kultur und damit der Landeskunde (vgl. Seidl 2002: 90) eines Landes ist Musik relativ leicht zugänglich: man hört sie im Radio, Liedtexte und Informationen über Musiker sind im Internet zu finden, viele städtische Bibliotheken bieten Musik-CDs an. Mit Musik können alle Fertigkeiten trainiert werden und kreative Methoden zum Einsatz kommen, durch die die Lerner offene Herangehensweisen an verschiedenste Fragestellungen einüben. Das sind einige der Gründe, die für den Einsatz von Liedern und Musik im DaF-Unterricht sprechen. Von den vielen Kriterien, die bei der Auswahl der Lieder beachtet werden sollten (vgl. Geiger 2002: 103 ff.; Rodriguez Cemillian 2000), stehen für die hier präsentierten Lehrmaterialien insbesondere praktische Erwägungen im Vordergrund: besonders die für Anfänger ausgewählten Lieder sind am Sprachniveau ausgerichtet und nach der Verständlichkeit sowohl des Liedtextes und als auch der Musiker ausgesucht. Ein weiterer praktischer Aspekt bei der Auswahl besteht in der Möglichkeit, die Lieder zu didaktisieren und interessante Übungen damit zu gestalten. Auch sind die landeskundlichen Themen der Liedtexte ein Auswahlkriterium. Schließlich sind die
Lieder auch nach dem Kriterium des persönlichen Geschmacks ausgewählt (worüber sich dann sicherlich streiten läßt). Da bei der Arbeit mit Musik ein nicht zu unterschätzendes emotionales Element in den Unterricht einfließt, wird der Unterricht mit Liedern im allgemeinen authentischer, wenn man sich mit der Musik identifiziert.

\section{Lehrmaterialien}

Die folgenden Lehrmaterialien sind mehrfach mit Lerngruppen von etwa 20 Teilnehmern aus verschiedensten Ländern im Alter zwischen 18-30 Jahren erprobt. Die Lehrmaterialien sind wie folgt strukturiert: als Überschrift wird der Name der Gruppe oder der Band genannt und dazu der Titel des bearbeiteten Liedes. Zunächst wird die Musikgruppe kurz vorgestellt und die Auswahl des Liedes erläutert. Dann wird der Liedtext aufgeführt. Der Schwerpunkt der Lernziele bezeichnet die einzuübende Fertigkeit oder den Lerninhalt. Die angewandten Methoden werden kurz erklärt und können so auf andere Lieder übertragen werden. Der Unterrichtsverlauf ist in Schritte unterteilt, wobei zusätzliche Hinweise die Durchführbarkeit erleichtern sollen.

\subsection{Die Prinzen: Gabi und Klaus}

Die Prinzen veröffentlichten Gabi und Klaus 1991 als erstes Lied und seitdem ist die Band im deutschsprachigen Raum 
recht bekannt. Stilistisch können sie der Richtung Rock und Pop zugeordnet werden, viele ihrer Lieder haben Die Prinzen auch a capella gesungen. Ihre Texte haben amüsante und ironische, aber auch kritische Inhalte. Wie sich in zahlreichen Gesprächen mit DaF-Lehrern und -Lehrerinnen zeigte, sind die Prinzen nicht zuletzt wegen ihrer deutlichen Aussprache und guten Verständlichkeit relativ beliebt. Gabi und Klaus ist zudem in einer einfachen Satzstruktur verfaßt und daher für Anfänger geeignet. Interessant ist bei diesem Lied der Gegensatz zwischen Inhalt und Musik.

\section{Die Prinzen: Gabi und Klaus (Liedtext)}

Gabi hat Sehnsucht, Sehnsucht nach Klaus, doch Klaus sagt kühl nur: »Es ist aus.«. Gabi muß weinen, ihr Herz wird so schwer, doch Klaus sagt kühl nur: »Ich will dich nicht mehr.«.

Gabi ist einsam, schrecklich allein, sie denkt an Klaus und sagt sich: »Er ist so gemein! «.

Sie will ihn vergessen und damit das geschieht,

rennt sie durch den Regen und singt dieses Lied:

Das Leben ist grausam und schrecklich gemein.

Das Leben ist grausam und Klaus ist ein Schwein.

Doch plötzlich merkt Klaus, wie er Gabi liebt.

Er geht zu ihr, hofft, daß sie ihm vergibt.

Er steht vor ihr, wird vor Verlegenheit rot, doch sie sagt kühl nur: »Hau ab, du Idiot!«.

Klaus ist jetzt einsam, schrecklich allein, er denkt an Gabi und sagt sich: "Sie ist so gemein! «.

Er will sie vergessen und damit das geschieht,

rennt er durch den Regen und singt dieses Lied:

Das Leben ist grausam und schrecklich gemein.

Das Leben ist grausam und Gabi ein Schwein.
Schwerpunkt: Textproduktion

Methode: Kreatives Schreiben (vgl. Pommerin 1996: 11-19):

Bei dieser Methode dient der Liedtext zunächst als Schreibanlaß. Dabei sucht der Kursleiter aus dem Text etwa zehn bedeutungstragende oder schwierige Wörter bzw. Ausdrücke aus, mit deren Hilfe die Teilnehmer eine Geschichte schreiben sollen.

Zielgruppe: Anfänger nach 150-200 Unterrichtsstunden (A1-A2 entsprechend der Einteilung nach dem Europäischen Referenzrahmen)

\section{Unterrichtsverlauf:}

\section{Schritt 1:}

Folgende Wörter/Ausdrücke werden an die Tafel geschrieben und ihre Bedeutung geklärt:

Gabi und Klaus

Sehnsucht haben nach jdm. kühl

»Es ist aus. « gemein sein an jdn. denken jdn. vergessen einsam sein die Verlegenheit $j \mathrm{dm}$. vergeben "Das Leben ist grausam."

\section{Schritt 2:}

Die Arbeitsanweisung an die Kursteilnehmer lautet: Schreiben Sie eine Geschichte über/einen Dialog zwischen Gabi und Klaus mit diesen Wörtern/ Ausdrücken! Verwenden Sie mindestens sieben davon!

\section{Schritt 3:}

Die Kursteilnehmer bilden Arbeitsgruppen mit drei bis vier Personen und haben zur Lösung der Aufgabe $45 \mathrm{Mi}$ nuten Zeit.

Schritt 4:

Die Texte werden im Plenum vorgelesen. (Hinweis: $\mathrm{Zu}$ diesem Zeitpunkt möglichst keine Korrekturen mehr anbringen, da hier die Geschichten als 
Gesamttext im Vordergrund stehen sollen.)

\section{Schritt 5:}

Das Lied wird den Kursteilnehmern ohne Liedtext vorgespielt. Da die wichtigsten Wörter in Schritt 1 geklärt wurden, haben die Kursteilnehmer beim Hören kaum Schwierigkeiten. (Hinweis: Eine Bemerkung zu den im Lied verwendeten Schimpfwörtern ist hilfreich. Der Liedtext kann abschließend verteilt werden oder man kann das Lied einfach als Hörprobe wirken lassen.)

\subsection{Wir sind Helden: Guten Tag}

Wir sind Helden gehören $\mathrm{zu}$ den neuen Musikgruppen in Deutschland. 2002 veröffentlichten sie ihre ersten Lieder und Anfang 2004 wurden sie als beste Nachwuchsband ausgezeichnet. Der Stil ihrer Musik reicht von New Wave bis zu Rock und Pop. In ihrem Album Die Reklamation setzt sich die Gruppe einerseits kritisch mit unserer Gesellschaft auseinander, andererseits finden sich auf dem Album auch ironische Lieder. Das Lied Guten Tag könnte in der Tradition von »Protestsongs « stehen und bietet eine gute Grundlage für landeskundliche Arbeit.

\section{Wir sind Helden: Guten Tag (Liedtext)}

Meine Stimme gegen dein Mobiltelefon

Meine Fäuste gegen eure Nagelpflegelotion Meine Zähne gegen Dr. Best und seinen Sohn

Meine Seele gegen eure sanfte Epilation

Es war im Ausverkauf im Angebot die Sonderaktion

Tausche blödes altes Leben gegen neue Version

Ich hatte es kaum zu Hause ausprobiert, da wußte ich schon

an dem Produkt ist was kaputt - das ist die Reklamation.

Guten Tag, guten Tag, ich will mein Leben zurück
Ich tausche nicht mehr, ich will mein Leben zurück

Guten Tag, ich gebe zu, ich war am Anfang entzückt,

aber euer Leben zwickt und drückt nur dann nicht,

wenn man sich bückt

Guten Tag

Meine Stimme gegen die der ganzen Talkshownation

Meine Fäuste für ein müdes Halleluja und Bohnen

Meine Zähne gegen eure zahme Revolution Visionen gegen die totale Television

Es war im Ausverkauf im Angebot die Sonderaktion

Guten Tag, guten Tag, ich will mein Leben zurück...

Schwerpunkte: Hörverstehen und Leseverstehen, Landeskunde

Methoden:

Internetrecherche: über viele Gruppen gibt es Informationen im Internet. Diese Übung wird von den Kursteilnehmern außerhalb des Unterrichts vor der Arbeit mit dem Lied erledigt.

\section{Textpuzzle:}

Hier werden die Liedzeilen als Puzzleteile verwendet, indem man den Text in einzelne Zeilen zerschneidet.

Fragen zum Textverständnis

Zielgruppe: Mittelstufe-Fortgeschrittene (B1-C1).

\section{Unterrichtsverlauf:}

Vor dem Unterricht: Als Hausaufgabe wird eine Internetrecherche zur Band »Wir sind Helden« durchgeführt. Dabei sollen die Teilnehmer Informationen $\mathrm{zu}$ den Bandmitgliedern, ihren Themen und ihrem Werdegang suchen und ein kleines Profil der Band erstellen.

Schritt 1: (falls die Internetrecherche als Hausaufgabe gestellt wurde):

die Teilnehmer tauschen sich in Kleingruppen über die zur Band gefundenen Informationen aus. 


\section{Schritt 2:}

Vor dem Hören: Da das Lied einige schwierige Wörter enthält, müssen diese vorab geklärt werden. (Vorschlag für die zu klärenden Wörter: die Reklamation, Dr. Best, die Nagelpflegelotion, die Faust, der Ausverkauf, die Sonderaktion, das Angebot, sich bücken, zahm, die Seele). Zuerst sollte versucht werden, diese Wörter in Gruppenarbeit erschließen zu lassen. Anschließend können sie im Plenum besprochen werden. Schritt 3:

Die Kursteilnehmer erhalten in Zweieroder Dreiergruppen das Textpuzzle. Sie haben Zeit, die ungeordneten Textzeilen des Liedes kurz zu lesen.

\section{Schritt 4:}

Das Lied wird vorgespielt. Die Aufgabe lautet: »Bringen Sie die Zeilen des Liedes während des Hörens in die richtige Reihenfolge! «

Schritt 5:

Nach einer kurzen Besprechung der Kursteilnehmer untereinander wird das Lied ein zweites Mal vorgespielt und die fehlenden Zeilen werden zugeordnet. Normalerweise reichen zwei Durchgänge aus, um die richtige Reihenfolge zu ermitteln.

Schritt 6:

Zum Verständnis können folgende Fragen gestellt werden:

- »Die Sängerin des Liedes übt Kritik: Woran?«

- »Versuchen Sie den Ausdruck >Ich will mein Leben zurück` zu erklären!

\section{Schritt 7:}

Ein schriftlicher Kommentar und eine Diskussion bilden den Abschluß der Bearbeitung: »Wie gefällt Ihnen dieses Lied - inhaltlich sowie musikalisch? Begründen Sie Ihre Meinung, indem Sie eine Kritik zu dem Lied schreiben!« Hinweis: Hier kann man sich auf eine recht kontroverse Diskussion einstellen.

\subsection{Element of Crime: Immer unter Strom} Element of Crime wird häufig als »Kultband aus Berlin « bezeichnet. Diese Gruppe ist seit 20 Jahren in der deutschen Musikszene aktiv. In den ersten Jahren spielte die Gruppe englische Lieder und seit 1991 sind die Musikstücke in deutscher Sprache verfaßt. Stilistisch läßt sich ihre Musik dem Bereich Rock zuordnen. In ihren Texten beschäftigt sich die Band mit der heutigen Gesellschaft und ebenso mit persönlichen Themen. Das Lied Immer unterwegs handelt von der Mobilität und der Unruhe des heutigen Lebens.

Element of Crime: Immer unter Strom (Liedtext)

Im Gepäck nicht mehr als sieben Sachen Auf dem Kompaß nichts als geradeaus Und alle 100 Kilometer Helm ab zum Gebet Wegzukommen ist uns heilig

Anzukommen ist egal

Und die größte Qual ist uns die Angst, daß es mal nicht mehr weitergeht

Immer unter Strom

Immer unterwegs und überall zu spät

Was sich nicht bewegt ist nicht $\mathrm{zu}$ fassen

Wo wir sind wird uns der Boden heiß

Unsre Haut wird uns zur engen Hülle, die unter Spannung steht

Wo wir war(e)n war immer alles fade

Wo wir hinfahr(e)n wird es wunderbar

Und hoffen dürfen wir solange, wie sich der

Motor dreht

Immer unter Strom

Immer unterwegs und überall zu spät

Bring mich von hier weg

Bring mich dahin, wo noch irgendwas passiert

Bring mich von hier weg

Bring mich dahin, wo noch irgendetwas lebt

Bring mich von hier weg

Bring mich dahin, wo es anders ist, als hier Immer unter Strom

Immer unterwegs und überall zu spät

Bis nach Oslo sind es nur zwei Stunden Und nach Zürich höchstens zweieinhalb Und dennoch ist da immer etwas, das war schon vor uns da

Und etwas, das schon wieder weg ist Und etwas, das uns an den Hacken klebt 
Das uns bedroht, das uns verrät

Immer unter Strom

Immer unterwegs und überall zu spät

Schwerpunkte: mündliche Kommunikation und Leseverstehen

\section{Methoden:}

Bildbeschreibung: ein Foto der Band wird beschrieben. Beschreibung der Musik/Stimmung des Liedes. Arbeitsblatt mit Zuordnungsaufgaben und Fragen.

Zielgruppe: Mittelstufe - Fortgeschrittene (B1-C1)

\section{Unterrichtsverlauf:}

\section{Schritt 1:}

Als Einstieg dient ein Foto der Gruppe, das man beschreiben lassen kann (Fotos der Gruppe findet man im Internet). Das Foto sollte nicht vom Kursleiter kommentiert werden und die Fragen dazu lauten z. B.: Was sehen Sie? Wen? Wo? Beschreiben Sie Stimmung und Atmosphäre des Bildes! (Hinweis: Da Musiker auf Fotos häufig in einem bestimmten Stil dargestellt sind, erkennen zumeist einige Kursteilnehmer, daß auf dem Bild eine Musikgruppe gezeigt wird. Wenn das nicht der Fall ist, sollte vor dem Hören des Liedes die Verbindung zwischen dem Bild und der Musik hergestellt werden.

Schritt 2:

Das Instrumentalvorspiel des Liedes (30 Sekunden lang) hören. Fragen: Welche Stimmung beschreibt die Musik? Wie wirkt diese Musik auf Sie? Welche Instrumente hören Sie?

\section{Schritt 3:}

Der Titel des Liedes Immer unter Strom wird an die Tafel geschrieben und seine mögliche Bedeutung diskutiert.

\section{Schritt 4:}

Das Lied zusammen mit dem Text hören. (Hinweis: Da die Aussprache des Sängers in diesem Lied nicht besonders deutlich ist, wird empfohlen, das Lied nur bei sehr fortgeschrittenen Lernern ohne Vorgabe des Textes einzusetzen.) Schritt 5:

Das unten schematisch abgebildete Arbeitsblatt wird in Kleingruppen bearbeitet. Während dieser Arbeitsphase muß der Kursleiter möglicherweise einige im Lied vorkommende Wörter erklären. (Hinweis: Aufgabe 1 sollte zuerst bearbeitet und gleich anschließend im Plenum besprochen werden, da diese Aufgabe zur Klärung der Wortschatzfragen wichtig ist. Danach in Gruppenarbeit mit Aufgabe 2 fortfahren.)

\section{Arbeitsblatt zu »Immer unter Strom «}

1. Suchen Sie Wörter/Redewendungen im Lied, die folgenden Wortfeldern/ Themen zugeordnet werden können:

\begin{tabular}{l|l}
$\begin{array}{l}\text { Bewegung/un- } \\
\text { terwegs sein }\end{array}$ & $\begin{array}{l}\text { Stillstand/hier } \\
\text { sein }\end{array}$ \\
\hline &
\end{tabular}

2. Diskutieren Sie gemeinsam über die folgenden Fragen und notieren Sie in Stichwörtern:

2.1 Wer ist »wir«? Versuchen Sie Adjektive $\mathrm{zu}$ finden, die diese Menschen charakterisieren!

2.2 Beschreiben Sie die Lebenseinstellung, Lebenssituation (Alter, Beruf, wo wohnen sie...) dieser Menschen!

2.3 Stellen Sie Ihre persönliche Meinung zu dieser Lebenseinstellung dar! (Kennen Sie das hier beschriebene Gefühl von sich selbst, von anderen? Positive/negative Aspekte)

\subsection{Ganz schön feist: Immer wenn die Sonne scheint}

Seit etwa 10 Jahren singt die Gruppe Ganz schön feist freche und amüsante Lieder über den Alltag und seine Tücken. Ihren Stil definiert Ganz schön feist selbst als »Popacapellacomedy«. Die Sänger sind hauptsächlich dem interessierten Publi- 
kum bekannt. Das Lied Immer wenn die Sonne scheint beschreibt in einer verständlichen Alltagssprache das Thema "Schwimmbad in Deutschland « und spricht die Lerner wegen seines ironischfrechen Inhalts an.

Ganz Schön feist: Immer wenn die Sonne scheint (Liedtext)

35 Grad im Schatten

Wie lange wir das schon nicht mehr hatten

Ich stelle mich in der Schlange an

Weil ich es kaum erwarten kann

Es riecht nach Sonnenöl, Pommes und

Chlor

Und nirgendwo anders kommt so ein Geruch vor

Eine Affenhitze - Mitte Mai

Der Bademeister gibt den Zehner frei

Immer wenn die Sonne scheint

....

Wenn dann die Melodien über die Liegewiese wandern

Ein Ghettoblaster gegen den anderen

Je tätowierter der Besitzer ist

Desto toleranter bist du

Die liegen alle oben ohne, die liegen alle oben ohne da

Aber das stört mich nicht die Bohne

Ich finde das sowieso viel natürlicher

Immer wenn die Sonne scheint

....

Ich mache eine Ente vom Einer

Und so wie ich macht die keiner

Ich mache einen »Köpper « vom Zehner

Aber die Ente war schöner

Kleine Kinder müssen manchmal einfach

so

Und kleine Kinder gehen dazu nicht aufs

Klo

Im Kinderbecken war ich früher selber

$\mathrm{Da}$ ist das Wasser etwas gelber

Wie gut, daß ich im anderen Becken bin

Wie gut, wie gut...

Kinder sammeln Flaschenpfand

Alle springen vom Beckenrand

Wassereis schmilzt in der Hand

Majo ohne Pommes drauf

Wassereis ist ausverkauft

Frotteetuch mit Bärchen drauf

Der Tag geht zur Neige

In der Kabinen-Umkleide hat jemand fünfzig Mark verloren
Ich gucke, ob wer guckt

Schau mich um und warte

Dann hol ich mir davon eine Jahreskarte

Immer wenn die Sonne scheint

Schwerpunkte: Wortschatzübung und Hörverstehen

Methoden:

Assoziogramm zum Thema Schwimmbad

Hörverstehen: Der Liedtext wird als Lückentext gehört, wobei jeweils die Reime am Zeilenende weggelassen werden, um das Erkennen der fehlenden Wörter zu erleichtern.

Zielgruppe: Anfänger nach 250-300 Unterrichtstunden (A2)

Unterrichtsverlauf:

\section{Schritt 1:}

»Immer wenn die Sonne scheint...« an die Tafel schreiben. Die Kursteilnehmer schreiben den Satz zu Ende und lesen ihre Sätze vor.

\section{Schritt 2:}

In Deutschland denken viele Leute bei Wärme und Sonnenschein an einen Schwimmbadbesuch. Das wird zum Thema der ersten Gruppenarbeit: Die Teilnehmer erstellen in Kleingruppen ihr eigenes Assoziogramm zum Thema »Schwimmbad «.

\section{Schritt 3:}

Ein gemeinsames Assoziogramm wird mit den Kursteilnehmern und unter Benennung möglichst vieler Begriffe an der Tafel festgehalten. Dabei ergänzt der Kursleiter das Wortfeld »Schwimmbad « mit entsprechenden Wörtern aus dem Lied, falls diese nicht genannt werden. (Beispiele für solche Wörter aus dem Lied: Schwimmbecken, Frotteetuch, Zehner, Chlor, Bademeister, Liegewiese, Köpper, Wassereis, ...). (Hinweis: Man sollte die Kursteilnehmer vor oder nach dem Hören kurz auf umgangssprachliche Elemente im Lied hinweisen.) 


\section{Schritt 4:}

Das Lied wird zum ersten Mal gehört. Dabei wird der Lückentext mit Reimen ergänzt.

\section{Lückentext mit Reimen (Auszug)}

Ganz Schön feist: Immer wenn die Sonne scheint

35 Grad

Wie lange wir das schon nicht mehr hatten

Ich stelle mich in der

Weil ich es kaum erwarten kann

Es riecht nach Sonnenöl, Pommes und

Chlor

Und nirgendwo anders kommt so ein

\section{Eine Affenhitze - Mitte Mai}

Der Bademeister gibt

Immer wenn die Sonne scheint

\section{Schritt 5:}

Nachdem die Kursteilnehmer ihre Ergebnisse miteinander verglichen und sich ausgetauscht haben, wird das Lied noch einmal gehört und die restlichen Lücken werden ergänzt.

\section{Schritt 6:}

Hier einige Beispiele für Fragen, die mündlich in Gruppen und im Plenum besprochen werden können:

- Was gibt es alles in diesem Schwimmbad?

- Was haben Sie erwartet?/Was ist bekannt?

- Was ist unerwartet/überraschend?

- Im Lied werden die verschiedenen Sinneswahrnehmungen angesprochen: Nennen Sie die fünf Sinne und suchen Sie diese im Text!

- Was ist mit den »50 Mark « passiert?

- Erklären Sie den »Je-desto«-Satz!

- Suchen Sie umgangssprachliche Ausdrücke im Text!

- Wenn möglich, vergleichen Sie Baden und Schwimmen in Ihrer Heimat und in Deutschland!

\section{Weitere Ideen}

Wer sich mit deutschsprachiger Musik beschäftigt, dem fällt auf, daß die Medien englischsprachige Musik meist bevorzugen und es daher nicht immer leicht ist, deutsche Lieder zu finden. Deshalb werden hier einige mögliche Quellen genannt. Neben den oben bereits erwähnten Stadtbibliotheken ist das Internet eine reiche Fundgrube. Vielfältige Angaben hierzu finden sich im Internet-Dossier »Musik im Unterricht" von Andreas Westhofen (siehe Literaturliste)

In der Liederbestenliste (www.liederbestenliste.de) veröffentlichen Mitarbeiter verschiedener Radiosender jeden Monat eine Auswahl von Liedern in deutscher Sprache. Schließlich ist als Radiosender, der nicht nur englische, sondern auch deutschsprachige Musik spielt, das Deutschlandradio Berlin $\mathrm{zu}$ empfehlen (nähere Informationen unter www.dradio.de).

Abschließend wird noch einmal auf die Auswahlkriterien eingegangen. Wenn man in einem Kurs mehrere Lieder einsetzt, ist es interessant, verschiedene $\mathrm{Mu}$ sikstile und ganz unterschiedliche Musiker zu wählen, damit die Teilnehmer einen ersten Einblick in die Vielfältigkeit der Liederlandschaft gewinnen. Dabei ist ein interkultureller Vergleich verschiedener Musikstile ein bereicherndes Thema. Die Auswahl nach landeskundlichen Aspekten kann ebenso der Weg zum Einsatz von Liedern sein: Wenn man seine Liedersammlung nach ganz allgemeinen Themen (wie Mensch - Gesellschaft, Männer - Frauen, Stadt - Land, Gefühle, Natur, Freundschaft usw.) durchsucht, kann man Ideen für Unterrichtsreihen oder für Verbindungen zwischen einzelnen Liedern finden. 


\section{Literatur}

Geiger, Klaus: »Musik im Literatur- und Landeskundeunterricht«, Mitte. Zeitschrift für interkulturelle Begegnung 1 (2002), 103-113.

Goethe-Institut Inter Nationes u. a. (Hrsg.): Europarat - Rat für kulturelle Zusammenarbeit: Gemeinsamer europäischer Referenzrahmen für Sprachen: lernen, lehren, beurteilen. Berlin; München: Langenscheidt, 2001.

Pool-Lifdu (Hrsg.): Mein Gespräch, meine Lieder. Berlin; München: Langenscheidt, 1986.

Pool-Lifdu (Hrsg.): Heute hier, morgen dort. Berlin; München: Langenscheidt, 1991.

Pommerin, Gabriele: Tanzen die Wörter in meinem Kopf. Kreatives Schreiben für den DaF-Unterricht. Ismaning: Hueber, 1996.

Rodriguez Cemillian, Dolores: Internet Dossier: Musik im Unterricht (Teil 3). www.deutsch-als-fremdsprache.de/infodienst/2000/daf-info1-00.php3.

Seidl, Christian: »Einsatz deutscher Rockmusik für interkulturelle Landeskunde im Bereich Deutsch als Fremdsprache", Mitte. Zeitschrift für interkulturelle Begegnung 1 (2002), 90-102.

Westhofen, Andreas: Internet Dossier: Musik im Unterricht (Teil 1-2). www.deutsch-alsfremdsprache.de/infodienst/1999/dafinfo11-99.php3.

\section{Verzeichnis der verwendeten Musik}

Element of Crime: Weißes Papier. Polydor, 1993.

Ganz schön feist: In echt. Roof Music, 2001.

Die Prinzen: A-Capella-Album! BMG Ariola Miller, 1997.

Wir sind Helden: Die Reklamation. EMI-Music, 2003. 